\title{
Does increased ICU strain prompt clinicians to communicate with families about end-of-life issues earlier?
}

\author{
Renee D. Stapleton ${ }^{1 *}$ and Dominique D. Benoit ${ }^{2}$
}

(C) 2016 Springer-Verlag Berlin Heidelberg and ESICM

The concept of intensive care unit (ICU) strain-the collision of sick patients, frequent emergencies, tense emotions, and death's uncertainty in a setting with constrained resources and time-is perennial. Those who care for critically ill patients in busy ICUs feel this strain viscerally and sense that it worsens clinician morale, makes us feel less efficient and satisfied, reduces quality of trainee education, and may negatively impact patient care. However, while the notion of ICU strain is not new, operationalizing this concept to allow its investigation has only recently emerged.

In this issue of Intensive Care Medicine, Hua and colleagues [1] examine the association of ICU strain during the first 3 days of ICU admission (measured by ICU census, proportion of new admissions comprising the daily census, and acuity of illness) and timing of do not resuscitate (DNR) orders and death. In a retrospective cohort study of ICU decedents using Project IMPACT data [2], they report that among 9066 patients with any therapy limitation in place, some measures of ICU strain are significantly associated with reduced time to DNR orders and shorter time to death. The effect of strain on time to death was nearly all mediated by reduced time to DNR, suggesting that patients died sooner as a result of earlier decisions to limit life-sustaining therapy. Among the 825 patients without limitations in therapy, there was no association between strain and time to death.

This same research group has studied ICU strain for several years. In 2011, Dr. Halpern described a metric of ICU strain and its utility in outcomes research

\footnotetext{
*Correspondence: renee.stapleton@uvm.edu

1 Pulmonary and Critical Care Medicine, University of Vermont, Burlington, VT, USA

Full author information is available at the end of the article
}

[3]. Further work followed correlating the metric with ICU physicians' and nurses' perceptions of strain [4]. Additional studies utilizing Project IMPACT data have investigated how increased ICU strain might influence patient outcomes and clinician behavior (Table 1). This body of work has produced several important results. First, increased ICU strain at admission is associated with increased hospital death in closed ICUs (odds ratio [OR] 1.07, 95 \% CI 1.01-1.11) but not open ICUs (OR 1.01, $95 \%$ CI 0.99-1.03) [5]. Second, increased strain at ICU discharge is associated with decreased length of ICU stay and increased risk of ICU readmission, but not with subsequent in-hospital death, hospital length of stay, or discharge disposition [6]. Third, more strain is associated with less adherence to venous thromboembolism prophylaxis guidelines [7]. Finally, in a single-center study, increased ICU strain is also associated with more overall time spent on daily rounds, but less time spent per patient rounding on new admissions [8].

Beyond guideline adherence and rounding time, this new study suggests that increased ICU strain may change another facet of clinician behavior-that of decision-making and communication with families. One plausible explanation mentioned by the authors is that increased strain prompts clinicians to engage in end-of-life discussions earlier than during times of less strain. End-of-life communication can be difficult. It takes time and emotional effort, and is easy to deprioritize. Perhaps, under more pressure working in the ICU, we begin necessary communication earlier. Maybe we are more efficient at doing what needs to be done and more quickly communicate with families of patients likely to die regardless of when these discussions take place. Although conceivable, this reasoning alone does not explain the prior study's findings (using the same

\section{伨


Table 1 Summary of results from prior publications

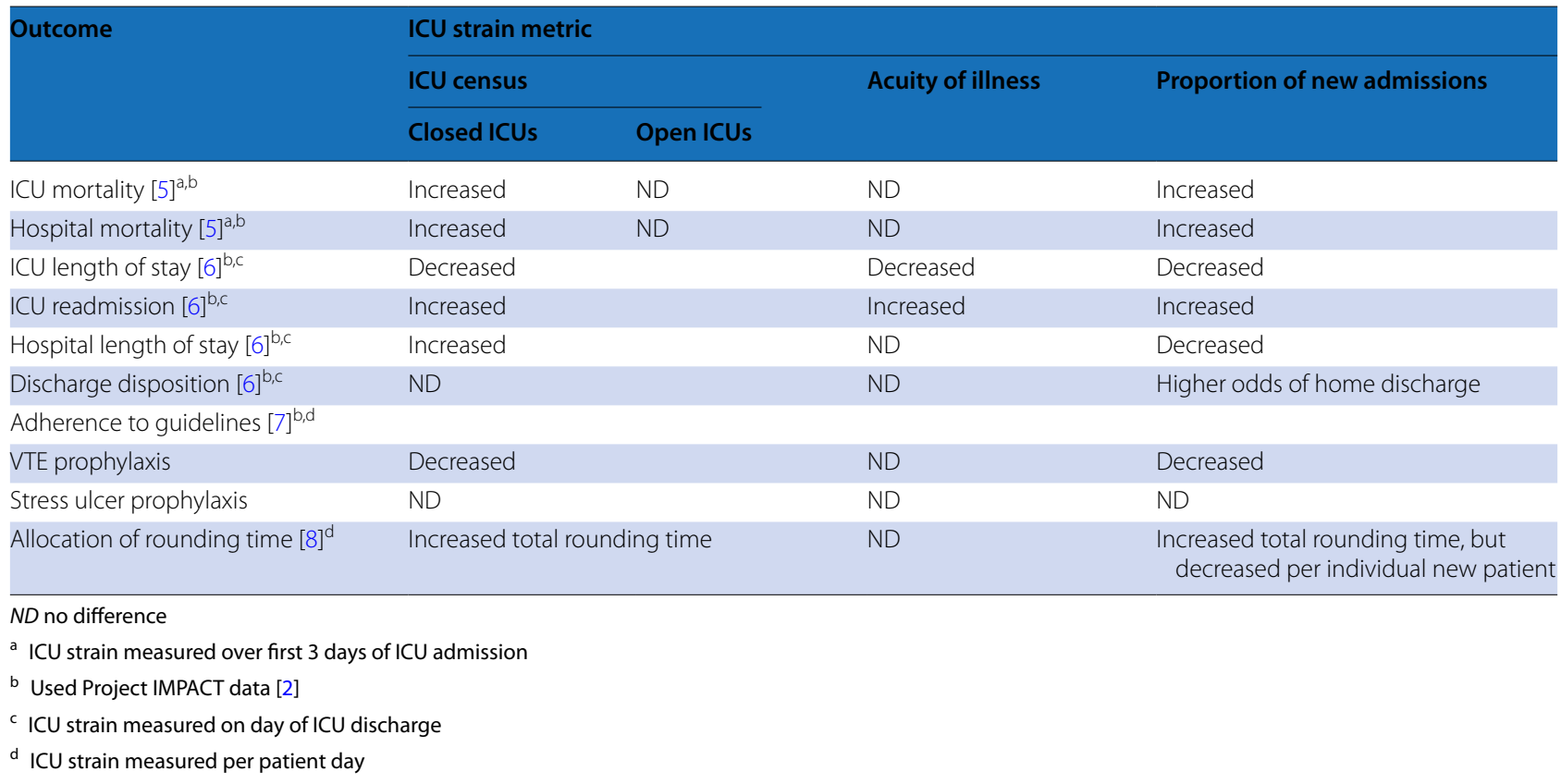

data) that ICU and hospital mortality were increased for patients admitted to closed ICUs during time of more strain [5]. If improved clinician efficiency under increased strain leads to earlier limitations of life-sustaining therapy and death in patients destined to die, overall hospital mortality should not increase, unless a portion of those deaths would have occurred post-hospitalization in less strained times and therefore would not be captured as hospital deaths in the dataset. Another explanation is that increased ICU strain compromises patient care resulting in hastened clinical deterioration, and thus earlier limitations in therapy death. The authors propose and then refute this idea, on the basis that no association with ICU strain and death was seen in the 825 patients without care limitations. With their prior publication finding numerically small but statistically significant increases in ICU and hospital mortality during times of increased ICU strain [5], we wonder if the current study is underpowered (small sample size of group without therapy limitations) to detect small differences in mortality and, therefore, the results reflect a type II error in this population.

There are limitations to this study. Project IMPACT data are more than 7 years old; although it is unlikely, communication processes could have changed in the interim. Additionally, the study's definition of ICU strain is limited to measures of census, acuity, and new admissions; although this metric correlates with provider perceptions [4], it may not fully encompass the breadth of strain. Other factors such as inadequate staffing, trainee aptitude, and sick patients elsewhere in the hospital (e.g., emergency department) that consume ICU clinician time may also affect overall ICU strain, but are not included in the current definition. Finally, in this study DNR orders and death occurred approximately 6-8 days and 7-9 days, respectively, after ICU admission. ICU strain was measured over the first 3 ICU days, and it is possible that strain closer to the time of DNR orders and death is also relevant.

Even with its limitations, this interesting study provides additional insight into ICU clinician behavior and prompts new questions. Does increased ICU strain indeed have both negative and positive impacts? If so, how do we decrease strain to reduce pressure on clinicians, but at the same time encourage efficiency equivalent to times of increased strain? What is it about strain specifically that affects clinician behavior change? Is the content of ICU communication during times of increased strain different than that during less strain (i.e., perhaps clinicians are communicating early, but the adequacy of that communication is reduced under strain)? What other factors beyond ICU strain not related to patients themselves influence clinicians' communication and decision-making? In addition to inspiring further research, this new study should also encourage ICU clinicians to reflect on our motivations for decision-making about our patients and communicating with their loved ones. 


\section{Author details}

1 Pulmonary and Critical Care Medicine, University of Vermont, Burlington, VT, USA. ${ }^{2}$ Department of Intensive Care, Ghent University Hospital, Ghent, Belgium.

\section{Compliance with ethical standards}

\section{Conflicts of interest}

On behalf of all authors, the corresponding author states that there is no conflict of interest.

Received: 24 February 2016 Accepted: 6 March 2016

Published online: 20 April 2016

\section{References}

1. Hua M, Halpern SD, Gabler NB, Wunsch H (2016) Effect of ICU strain on timing of limitations in life-sustaining therapy and death. Intensive Care Med. doi:10.1007/s00134-016-4240-8

2. Cook SF, Visscher WA, Hobbs CL, Williams RL, Project ICIC (2002) Project IMPACT: results from a pilot validity study of a new observational database. Crit Care Med 30(12):2765-2770
3. Halpern SD (2011) ICU capacity strain and the quality and allocation of critical care. Curr Opin Crit Care 17(6):648-657

4. Kerlin MP, Harhay MO, Vranas KC et al (2014) Objective factors associated with physicians' and nurses' perceptions of intensive care unit capacity strain. Ann Am Thoracic Soc 11(2):167-172

5. Gabler NB, Ratcliffe SJ, Wagner J et al (2013) Mortality among patients admitted to strained intensive care units. Am J Respir Crit Care Med 188(7):800-806

6. Wagner J, Gabler NB, Ratcliffe SJ et al (2013) Outcomes among patients discharged from busy intensive care units. Ann Intern Med 159(7):447-455

7. Weissman GE, Gabler NB, Brown SE, Halpern SD (2015) Intensive care unit capacity strain and adherence to prophylaxis guidelines. J Crit Care 30(6):1303-1309

8. Brown SE, Rey MM, Pardo D et al (2014) The allocation of intensivists' rounding time under conditions of intensive care unit capacity strain. Am J Respir Crit Care Med 190(7):831-834 